\title{
BMJ Open How reliable is perioperative anticoagulant management? Determining guideline compliance and practice variation by a retrospective patient record review
}

\author{
Marco J Moesker, ${ }^{1}$ Janke F de Groot, ${ }^{2}$ Nikki L Damen, ${ }^{3}$ Menno V Huisman, ${ }^{4}$ \\ Martine $\mathrm{C}$ de Bruijne, ${ }^{1}$ Cordula Wagner ${ }^{1,2}$
}

To cite: Moesker MJ, de Groot JF, Damen NL, et al. How reliable is perioperative anticoagulant management? Determining guideline compliance and practice variation by a retrospective patient record review. BMJ Open 2019;9:e029879. doi:10.1136/ bmjopen-2019-029879

- Prepublication history and additional material for this paper are available online. To view these files, please visit the journal online (http://dx.doi. org/10.1136/bmjopen-2019029879).

Received 15 February 2019 Revised 17 June 2019 Accepted 24 June 2019

Check for updates

(C) Author(s) (or their employer(s)) 2019. Re-use permitted under CC BY-NC. No commercial re-use. See rights and permissions. Published by BMJ.

For numbered affiliations see end of article.

Correspondence to

Mr Marco J Moesker;

m.moesker@amsterdamumc.nl

\section{ABSTRACT}

Objectives Surgery in patients on anticoagulants requires careful monitoring and risk assessment to prevent harm. Required interruptions of anticoagulants and deciding whether to use bridging anticoagulation add further complexity. This process, known as perioperative anticoagulant management (PAM), is optimised by using guidelines. Optimal PAM prevents thromboembolic and bleeding complications. The purpose of this study was to assess the reliability of PAM practice in Dutch hospitals. Additionally, the variations between hospitals and different bridging dosages were studied.

Design A multicentre retrospective patient record review. Setting and participants Records from 268 patients using vitamin-K antagonist (VKA) anticoagulants who underwent surgery in a representative random sample of 13 Dutch hospitals were reviewed, 259 were analysed.

Primary and secondary outcome measures Our primary outcome measure was the reliability of PAM expressed as the percentage of patients receiving guideline compliant care. Seven PAM steps were included. Secondary outcome measures included different bridging dosages used and an analysis of practice variation on the hospital level.

Results Preoperative compliance was lowest for timely VKA interruptions: $58.8 \%(95 \% \mathrm{Cl} 50.0 \%$ to $67.7 \%)$ and highest for timely preoperative assessments: $81 \%$ (95\% Cl $75.0 \%$ to $86.5 \%)$. Postoperative compliance was lowest for timely VKA restarts: $39.9 \%(95 \% \mathrm{Cl} 33.1 \%$ to 46.7\%) and highest for the decision to apply bridging: $68.5 \%$ (95\% Cl $62.3 \%$ to $74.8 \%$ ). Variation in compliance between hospitals was present for the timely preoperative assessment (range $41 \%-100 \%$ ), international normalised ratio testing (range 21\%-94\%) and postoperative bridging (range 20\%-88\%). Subtherapeutic bridging was used in $50.5 \%$ of patients and increased with patients' weight. Conclusions Unsatisfying compliance for most PAM steps, reflect suboptimal reliability of PAM. Furthermore, the hospital performance varied. This increases the risk for adverse events, warranting quality improvement. The development of process measures can help but will be complicated by the availability of a strong supporting evidence base and integrated care delivery regarding PAM.
Strengths and limitations of this study

- We used a multicentre retrospective patient record review to assess the quality of perioperative anticoagulant care.

- This allowed us to evaluate the 'real-world' practice without risking bias by study setting or observations.

- Dependency of routinely recorded patient data had its own limitations, especially regarding the quality of preoperatively recorded perioperative anticoagulant management care.

- Some selection bias at the hospital level might have occurred due to the voluntary nature of participation.

- Clinical outcomes were not included in our assessment, preventing the establishment of a relationship with observed practice patterns.

\section{BACKGROUND}

Anticoagulant therapy is effective in preventing arterial thromboembolisms, including cerebral stroke, in patients with atrial fibrillation or a mechanical heart valves as well as preventing venous thromboembolism. Managing anticoagulant therapy is challenging for various reasons. Among these are the narrow therapeutic target ranges for the international normalised ratio (INR), susceptibility to dietary fluctuations and comedication interactions altering the anticoagulant intensity. ${ }^{12}$ This urges careful risk assessments and monitoring of anticoagulants to prevent adverse bleeding and thromboembolic events, ${ }^{34}$ both having a potential harmful, everlasting effect on quality of life in a largely elderly patient population. However, in the past decade, anticoagulants were identified as having one of the highest occurrence of medication-related adverse events. ${ }^{5-8}$ Corresponding with the first step in the 'safety measurement 
and monitoring' framework as proposed by Vincent et al, these studies confirm that anticoagulants cause harm, jeopardising patient safety. ${ }^{9}$ Consecutive quality improvement initiatives targeted at anticoagulant drugs are there for warranted, and some have already been undertaken. ${ }^{10-12}$

To inform these quality improvement efforts, this framework supports assessing the reliability, defined as 'failure-free operation over time', of standardised clinical systems and processes within healthcare. It applies to processes that healthcare professionals have to carry out reliably. ${ }^{9}$

Anticoagulant management around surgery can be regarded as such a process since international guidelines advise and assist standardisation. ${ }^{13}$ Surgery itself accounts for $21 \%$ of anticoagulant-related medication errors as found by Henriksen et al. ${ }^{14}$ Therefore, making it a relevant process for a reliability assessment.

Surgical procedures in anticoagulated patients require specific attention. While reducing the risk of thromboembolic events, uninterrupted anticoagulation increases bleeding risk during and after surgery. ${ }^{15} 16$ Hence, preoperative interruption of anticoagulation is often required. ${ }^{17}$ For a select group of high-risk patients, short-acting heparins, for example, low-molecular-weight heparins (LMWHs), are required during the interruption period to reduce the time at risk for thromboembolic complications. This is known as 'bridging anticoagulation' and is considered an off-label use of heparins without consensus on optimal dosing. ${ }^{13} 1819$ The process of managing anticoagulants around surgery is referred to as "perioperative anticoagulant management' (PAM). PAM entails several steps that healthcare professionals should carry out reliably to minimise thromboembolic and bleeding complications. ${ }^{20-22}$

Standardised PAM has been evaluated within study contexts several times and found that it was feasible and associated with a low risk for complications. ${ }^{19}{ }^{21-23}$ However, the persistent occurrence of anticoagulant-related adverse events around the time of surgery, questions the reliability of everyday PAM practice compared with study settings.

Evaluating the reliability of standardised PAM in everyday practice can reveal provider or process vulnerabilities that can help in interpreting adverse events. Studies evaluating PAM in everyday practice are scarce and have limited generalisability due to self-reported PAM practices by physicians, restricted patient populations and single centre based studies. ${ }^{24-27}$

Therefore, as the next step in safety measurement and monitoring, the primary aim of this study was to assess the reliability of everyday PAM practice from planning to patient discharge in a selection of Dutch hospitals. Reliability was assessed by determining the per cent of patients receiving guideline compliant care. Our secondary aims were to determine if PAM practice varied between hospitals and which heparin dosages are used for bridging anticoagulation.

\section{METHODS}

\section{Study setting, design and participants}

Long-term oral anticoagulant care in the Netherlands is characterised by a network of anticoagulant management services (AMS). These specialised services are responsible for monitoring and dosage of vitamin-K antagonist (VKA) anticoagulation outside the hospital setting. During hospitalisation, this responsibility is temporarily transferred from the AMS to the medical specialist until discharge. Also in case of PAM, a transfer of responsibility takes place. According to a Dutch integrated care standard, the surgeon together with the anaesthesiologist set the PAM policy. ${ }^{28}$ However, the responsibility for executing the PAM policy depends on whether or not the patient is admitted in the hospital. In case of admitted patients, the surgeon is responsible for PAM execution. If patients reside at home, the AMS are responsible. The PAM evaluation in this study was performed from the perspective from the hospital where surgery took place.

Hospitals were invited for participation after being selected through a random sampling procedure that was stratified for hospital type and geographical location (urban/rural). Participation was voluntary. At first, 19 hospitals were invited of which 10 agreed to participate. To improve the representativeness of our hospital sample relative to all Dutch hospitals, we sampled and invited an additional six hospitals of which three agreed to participate. In total, two university, four tertiary teaching and seven general hospitals participated (online supplementary figure 1). When given, official reasons for non-participation were: migrations between electronic health records, staffing shortages for facilitating the research and internal reorganisations.

Twenty records of patients using VKA admitted for surgery between 1 June 2015 and 31 December 2015 were randomly selected and reviewed. Randomisation of eligible patient records was performed by hospital or research personnel using a random number generator available in the local spreadsheet application. Patients were only included once per hospital. Inclusion criteria were: age $\geq 18$ years, length of stay $\geq 24$ hours, underwent acute or elective surgical procedure using general and/ or spinal/epidural anaesthesia. Exclusion criteria were: psychiatric or gynaecological/obstetric ward admission, admission from or discharge to other hospitals, trauma other than hip fractures on admission, pregnancy or 6 weeks postpartum and palliative care admission. In case of an irretrievable required (section of a) health record, a replacement record was randomly selected. This study focusses only on patients with interrupted VKAs. Therefore, we excluded records from analysis if the PAM policy was not recorded or when the VKA was not interrupted (online supplementary figure 1 ).

\section{Reliability assessment of PAM}

A panel of five experts in the thrombosis and haemostasis field was consulted throughout this study. The panel was involved in both developing case report forms 
and classification models. We distinguished seven steps in the PAM process for the reliability assessment. Based on guidelines and previous assessments, these steps were seen as critical to a safe execution of PAM:

Step 1 Timing of preoperative patient assessment

Step 2 Preoperative VKA interruption interval

Step 3 Preoperative INR testing

Step 4 Preoperative use of bridging anticoagulation

Step 5 Postoperative use of bridging anticoagulation

Step 6 Postoperative restart time for bridging anticoagulation

Step 7 Postoperative restart time for VKA

Reliability of individual PAM steps was defined as the percentage of patients receiving guideline compliant care. The adequacy of the decision to interrupt the VKA is not subject to evaluation in this study, because of the absence of a validated instrument to determine surgical bleeding risk.

Several relevant guidelines on PAM are available. During our data collection period in 2015, the Dutch Quality Institute for Healthcare (CBO) guideline for Diagnostics, Prevention and Treatment of Venous Thromboembolism and Secondary Prevention of Arterial Thrombosis released in 2008 was the leading PAM guideline. ${ }^{29}$ This guideline is an adaption of the American College of Chest Physicians (ACCP) guideline for warfarin patients released in $2004 .{ }^{30}$ However, it became apparent that in 2015 the $\mathrm{CBO}$ guideline more than likely reflected outdated evidence regarding PAM and current practice has since moved on. Especially since the ACCP updated its guideline in 2012. To account for this, we employed a hybrid frame of reference of the 2008 and 2012 guideline in assessing PAM reliability. We ensured that our assessment criteria reflected the pharmacological properties of acenocoumarol and phenprocoumon used in the Netherlands as opposed to warfarin used internationally. Table 1 provides an overview of all PAM steps, assessment criteria and sources used.

\section{Patient record review}

Trained research assistants and one researcher (MM) reviewed and extracted the data in all hospitals through standardised case report forms. Both outpatient preoperative patient assessment records and inpatient admission records were retrieved. Data extracted from the preoperative patient assessment records included the planned preoperative anticoagulant management, that is, whether the VKA was to be interrupted with or without bridging anticoagulation, interruption dates and bridging anticoagulation orders. Data required for the determination of thromboembolic risk were extracted from the medical history records. This included the indication for VKA use and presence of relevant comorbidities. Data extracted from inpatient admission records were INR test results, VKA and heparins administration and discharge orders.
Furthermore, general demographic, admission and surgery characteristics were extracted. The informed consent was waived because of the use of patient record data only (protocol number: 2015/430).

\section{Primary outcome measures: compliance of PAM}

Classification models were constructed for determining guideline compliance for each PAM step. Compliance for steps 1-4 was not assessed for patients undergoing non-elective or emergency surgery. Steps 5-7 were assessed for all patients while differentiating between elective and non-elective patients. All PAM evaluation criteria and the distinction between compliance and non-compliance are summarised in table 1 . We will discuss several steps in detail.

Steps 4 and 5 involve determining the thromboembolic risk in order to evaluate the appropriateness of bridging anticoagulation. Thromboembolic risk was determined based on the ACCP 2012 guideline and is provided in online supplementary table 1 . The classification of preoperative bridging anticoagulation was based on patient record annotations indicating towards the use of bridging anticoagulation. Postoperative use of bridging was analysed in more detail and included the dosage. Therapeutic and subtherapeutic dosed heparins were both classified as bridging (online supplementary table 2). A minimum of two consecutive days of bridging or a discharge prescription present was required for a classification of postoperative bridging. The adequacy of the duration of bridging therapy was not subject to evaluation.

Step 6 involves the postoperative restart time for bridging anticoagulation. Based on the CBO recommendations, postoperative bridging anticoagulation restarted on the first day following surgery was classified as compliant. CBO makes an exception for high thromboembolic risk patients, for which restarting bridging on the day of surgery is considered compliant as well.

\section{Secondary outcome measures: practice variation and bridging dosages}

Presence of practice variation between hospitals was analysed by determining the individual compliance per hospital. A minimum of 10 records per hospital per step was required to include the PAM step in variation analysis. The differences were statistically tested.

For the analysis of postoperative bridging dosages, a distinction was made between therapeutic and subtherapeutic dosages. Therapeutic dose heparins should be adjusted to the patient's weight.

Based on the patients' weight, bridging dosages were classified as either therapeutic or sub therapeutic using threshold values used in the Netherlands. ${ }^{31}$ Threshold values are provided in online supplementary table 2. Bridging dosage was determined per patient and the differences between patients were tested for elective and non-elective surgery, patients' thromboembolic risk and bodyweight strata. 
Table 1 Perioperative anticoagulant management (PAM) steps with evaluation criteria and source

\section{Preoperative}

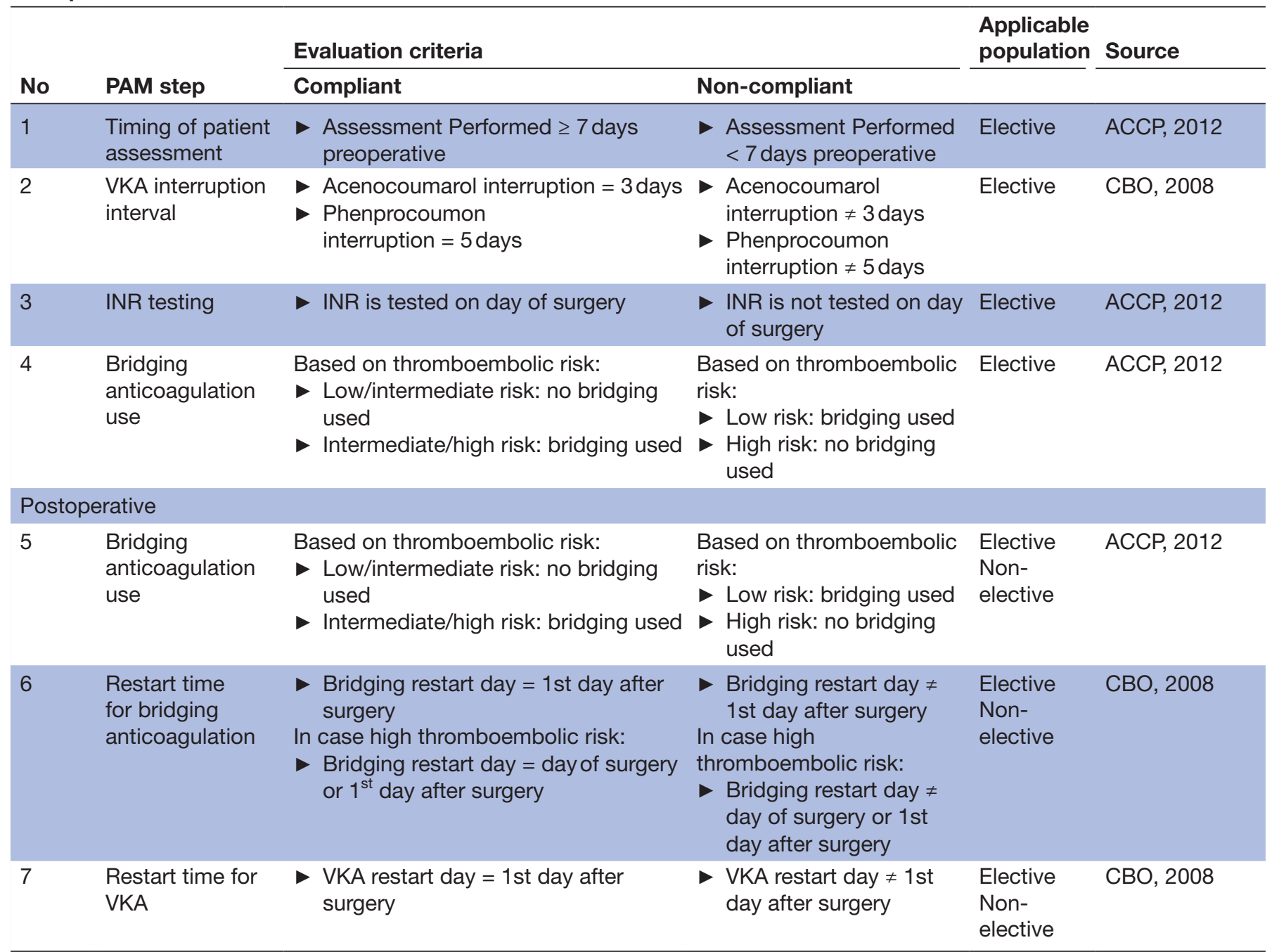

ACCP, American College of Chest Physicians; CBO, Dutch Quality Institute for Healthcare; INR, international normalised ratio; VKA, vitamin-K antagonist.

\section{Statistical methods}

To describe the study population and PAM practice characteristics, we used descriptive statistics. Compliance was expressed as the percentage of patients receiving guideline recommended care with $95 \%$ CIs. Differences in PAM practice characteristics and compliance between various groups were tested. Categorical outcomes were tested with $\mathrm{X}^{2}$ or Fisher's exact tests, continuous variables with the Man-Whitney U test due to skewness of the data.

Overall, practice variation between hospitals was tested with the $\mathrm{X}^{2}$ test or, when appropriate, the Fisher-Freeman-Halton exact test. Next, post hoc $\mathrm{X}^{2}$ tests between individual hospitals' performance for PAM steps were performed. For all tests, a $\mathrm{p}<0.05$ was considered statistically significant. Bonferroni correction was applied to control for type I error inflation during post hoc testing. Statistical analyses were performed with SPSS V.22 (IBM) and Stata V.14 (StataCorp).
Patient and public involvement

Patients and public were not directly involved in the current study.

\section{RESULTS}

\section{Study population}

We reviewed 268 patient records in 13 hospitals. Eleven hospitals used internal PAM protocols. The two remaining hospitals did not share information on protocol use. Nine records were excluded from analysis because of uninterrupted VKA or unclear recording of the PAM. The remaining 259 records (mean records per hospital=19.9; range=16-23) were analysed (online supplementary figure 1).

Demographic and clinical characteristics are displayed in table 2. The mean age of patients was 74.8 years $(\mathrm{SD}=10.6)$, most patients were male $(56.4 \%)$. Atrial 
Table 2 Demographic and clinical characteristics of included patients $(n=259)$

\begin{tabular}{|c|c|}
\hline Age (years), mean (SD) & $74.8(10.6)$ \\
\hline Male gender, n (\%) & $146(56.4)$ \\
\hline Elective surgery, n (\%) & $184(71.0)$ \\
\hline Length of stay (days), median (IQR) & $6.0(3.0-10.0)$ \\
\hline \multicolumn{2}{|l|}{ VKA, n (\%) } \\
\hline Acenocoumarol & 205 (79.2) \\
\hline Phenprocoumon & $54(20.8)$ \\
\hline \multicolumn{2}{|l|}{ Indication for VKA use, n (\%) } \\
\hline Atrial fibrillation & $173(66.8)$ \\
\hline Venous thromboembolism & $23(8.9)$ \\
\hline Mechanical heart valve & $9(3.5)$ \\
\hline Multiple $^{*}$ & $21(8.1)$ \\
\hline Other & $33(12.7)$ \\
\hline \multicolumn{2}{|l|}{ Comorbidities, n (\%) } \\
\hline iCVA/TIA & 37 (14.3) \\
\hline Thrombophilia & $7(2.7)$ \\
\hline Heart failure & $20(7.8)$ \\
\hline Hypertension & $132(51.0)$ \\
\hline Diabetes mellitus & $63(24.3)$ \\
\hline Active cancer/malignancy & $55(21.2)$ \\
\hline \multicolumn{2}{|l|}{ Thromboembolic risk, n (\%) } \\
\hline Low & $138(53.3)$ \\
\hline Moderate & $38(14.7)$ \\
\hline High & $40(15.4)$ \\
\hline Unknown† & $43(16.6)$ \\
\hline \multicolumn{2}{|l|}{ Type of surgical procedure, $n(\%)$} \\
\hline Gastrointestinal & $52(20.1)$ \\
\hline Orthopaedic & $89(34.4)$ \\
\hline Plastic & $3(1.2)$ \\
\hline Cardiac & $9(3.5)$ \\
\hline Neurosurgery & $5(1.9)$ \\
\hline Breast & $6(2.3)$ \\
\hline Vascular & $36(13.9)$ \\
\hline Urologic & 43 (16.6) \\
\hline Dental/ENT/HN & $2(0.8)$ \\
\hline Other & $14(5.4)$ \\
\hline
\end{tabular}

*Combination of two of the following indications: atrial fibrillation, venous thromboembolism and mechanical heart valve. †33 patients used VKA for other indications than AT9 provides TE-risk stratification, 10 patient records provided insufficient information to determine TE-risk.

ENT, ear, nose and throat; HN, head and neck.; iCVA, ischaemic cerebrovascular accident; TIA, transient ischaemic attack;

$\mathrm{TE}$, thromboembolic event; VKA, vitamin-K antagonist.

fibrillation was the most common indication for VKA use $(66.8 \%)$ followed by venous thromboembolism $(8.9 \%)$ and mechanical heart valve $(3.5 \%)$. Surgery was elective in $71.0 \%$ of patients; orthopaedic $(34.4 \%)$ and gastrointestinal $(20.1 \%)$ surgeries were most prevalent.

\section{Primary outcome measures: PAM practice and compliance}

The PAM practice characteristics and compliance with the guidelines are displayed in tables 3 and 4 . The median day of patient assessment (step 1) was 19 days preoperative ( $\mathrm{IQR}=8-37$ ) corresponding with a compliance of $80.8 \%$ with the recommended minimum of 7 days.

Data required for determining the duration of withholding VKA (step 2) were available in 119 (64.7\%) of elective patient records. Of these, VKA interruptions were compliant in $58.8 \%$ of patients. Acenocoumarol and phenprocoumon were interrupted for a median of 3 days $(\mathrm{IQR}=3-3)$ and 5 days $(\mathrm{IQR}=3-7)$, respectively.

Preoperative INR was tested (step 3) at the day of surgery in a majority of $60.9 \%$ elective patients, which is compliant with the recommendation. However, in $12.0 \%$, no recent INR test was performed.

Preoperative bridging policies (step 4) were available in 157 of the reviewed records (85.3\%). Among these, bridging was used in 47 (29.9\%). Preoperative bridging was used more frequently as the thromboembolic risk profile increased. Overall, preoperative bridging use was compliant in $79.7 \%$ of elective patients.

Postoperative bridging (step 5) was used in 107 patients $(41.3 \%$ ), with the highest bridging rate of $57.9 \%$ for moderate-risk patients. Postoperative bridging of low-risk patients differed between elective and non-elective patients $\left(29.4 \%\right.$ vs $48.5 \%$; $\left.\chi^{2} ; \mathrm{p}=0.044\right)$. Overall, the compliance of postoperative bridging was $68.5 \%$ and differed significantly between elective and non-elective patients $\left(73.5 \%\right.$ vs $\left.56.5 \% ; \chi^{2} ; \mathrm{p}=0.015\right)$.

Bridging was restarted (step 6) at a median of 1 day $(\mathrm{IQR}=0-1)$ whereas VKAs were restarted (step 7) at a median of 2 days $(\mathrm{IQR}=1-3)$ corresponding with compliances of $57.8 \%$ and $39.9 \%$, respectively. Both the bridging and VKA restart interval compliance did not differ between elective and non-elective patients $\left(\chi^{2} ; \mathrm{p}=0.42\right.$ and $\mathrm{p}=0.39$, respectively).

\section{Secondary outcome measures: practice variation and bridging dosages}

Variation between hospitals' compliance is displayed in figure 1. Performance varied significantly for the preoperative patient assessment $(\mathrm{p}<0.001)$, preoperative INR testing $(p<0.001)$ and postoperative use of bridging anticoagulation $(\mathrm{p}=0.001)$. Post hoc testing identified one significantly lower performing hospital for each of these PAM steps (hospitals 2 and 9) and one hospital performed significantly better with the INR testing (hospital 6).

Lastly, bridging dosages of LMWH were studied. Of the 107 bridged patients, $54(50.5 \%)$ received a subtherapeutic dosage, $45(42.1 \%)$ a therapeutic dosage, 4 $(3.7 \%)$ a combination and for another $4(3.7 \%)$ patients the distinction between the two dosages could not be established (results not shown in table). The bridging dosages did not vary between elective or non-elective 
Table 3 Perioperative anticoagulant management (PAM) practice characteristics and compliance for preoperative steps

\section{PAM step}

Elective surgery $(\mathrm{n}=184)$

1. Assess the patient at least 7 days before surgery

Valid records, n (\%) $182(98.9)$

Time from preoperative assessment to 19 (8-37)

surgery (days), median (IQR)

Compliance, \% $(95 \% \mathrm{Cl})$

80.8 (75.0 to 86.5$)$

\begin{tabular}{|c|c|}
\hline $\begin{array}{l}\text { 2. Preoperative VKA withholding durati } \\
\text { Acenocoumarol: } 3 \text { days } \\
\text { Phenprocoumon: } 5 \text { days }\end{array}$ & \\
\hline Valid records, n (\%) & $119(64.7)$ \\
\hline $\begin{array}{l}\text { Withholding duration acenocoumarol } \\
\text { (days), median (IQR) }\end{array}$ & $3(3-3)$ \\
\hline$<3$ days, $\mathrm{n}(\%)$ & $13(13.4)$ \\
\hline 3 days, $\mathrm{n}(\%)$ & $64(66.0)$ \\
\hline$>3$ days, $\mathrm{n}(\%)$ & $20(20.6)$ \\
\hline $\begin{array}{l}\text { Withholding duration phenprocoumon } \\
\text { (days), median (IQR) }\end{array}$ & $5(3-7)$ \\
\hline$<5$ days, $\mathrm{n}(\%)$ & $9(40.9)$ \\
\hline 5 days, $\mathrm{n}(\%)$ & $6(27.3)$ \\
\hline >5 days, n (\%) & $7(31.8)$ \\
\hline Compliance, \% (95\% Cl) & 58.8 (50.0 to 67.7$)$ \\
\hline
\end{tabular}

3. Test INR preoperative on the day of surgery

Valid records, n (\%) $184(100)$

Day of most recent preoperative INR

test, $\mathrm{n}(\%)$

\begin{tabular}{|c|c|}
\hline Surgery day & $112(60.9)$ \\
\hline Day before surgery & $50(27.2)$ \\
\hline Sooner/none & $22(12.0)$ \\
\hline $\begin{array}{l}\text { Preoperative INR on surgery day, } \\
\text { median (IQR) }\end{array}$ & $1.10(1.00-1.28)$ \\
\hline $\begin{array}{l}\text { Preoperative INR on day before } \\
\text { surgery, median (IQR) }\end{array}$ & $1.20(1.10-1.30)$ \\
\hline Compliance, \% (95\% Cl) & 60.9 (53.8 to 67.9$)$ \\
\hline \multicolumn{2}{|c|}{$\begin{array}{l}\text { 4. Apply or withhold preoperative bridging anticoagulation } \\
\text { according to thromboembolic risk }\end{array}$} \\
\hline Valid records, n (\%) & $157(85.3)$ \\
\hline \multicolumn{2}{|c|}{$\begin{array}{l}\text { Applied bridging per thromboembolic } \\
\text { risk strata, } \mathrm{n}(\%)\end{array}$} \\
\hline Low & $18(19.1)$ \\
\hline Moderate & $8(38.1)$ \\
\hline High & $9(50.0)$ \\
\hline Unknown & $12(50.0)$ \\
\hline Compliance, \% $(95 \% \mathrm{Cl})^{\star}$ & 79.7 (72.9 to 86.5$)$ \\
\hline
\end{tabular}

Unless otherwise stated, all results are based on valid records only. *Based on valid records and records with known thromboembolic risk.

INR, international normalised ratio; VKA, vitamin-K antagonist.

patients $\left(\chi^{2} ; \mathrm{p}=0.30\right)$ and for different thromboembolic risk strata $\left(\chi^{2} ; \mathrm{p}=0.39\right)$. However, bridging dosages varied between patient weight groups (Fisher's exact; $p<0.001$ ).
Subtherapeutic bridging dosage use increased as the patient weight increased (online supplementary figure $3)$.

\section{DISCUSSION}

This study aimed to assess the reliability of PAM in everyday, also referred to as 'real-world' practice in a sample of Dutch hospitals. Deviations from recommended PAM care were common. Depending on the PAM step of interest, deviations occurred in at least $19 \%$ of patients to as much as $60 \%$ of patients.

\section{Compliance and possible implications for practice}

The highest non-compliance was found for the VKA and bridging anticoagulation time of restart (steps 6 and 7). Both should be restarted after 24 hours postsurgery. However, the restart of bridging was premature or delayed in $42.2 \%$ of patients whereas VKA restart interval deviations were even more common, occurring in $60.1 \%$ of patients. The majority of these were attributable to a delayed restart, which is similar to the findings of others. ${ }^{24}$ Delayed restarts prolong the time patients are at risk for a thromboembolic complication due to suboptimal anticoagulation.

The reason for this low compliance for restart intervals is unclear. Restart postponement is preferred if adequate postsurgical haemostasis has not yet occurred; with the current study design, we could not observe the adequacy of this decision. However, allowing a 24-hour restart postponement would increase the compliance figures with a modest $8.7 \%$ and $13.1 \%$ for bridging and VKA restart, respectively. Another explanation, described by Flaker $e t a l$, is a difference in attitudes of clinicians in averting thromboembolic or bleeding complications. Clinicians with a risk-averse attitude towards bleeding complications might favour a delayed restart, whereas a risk-averse attitude towards thromboembolic complications might result in a premature restart. ${ }^{32}$

Also, preoperatively the timing of VKA interruption (step 2) appears to be troublesome. An inadequate interruption interval exposes patients to a prolonged thromboembolic risk in case of preliminary VKA interruption. Alternatively, delays or cancellations of surgery can occur in case of delayed VKA interruptions. These situations should be prevented as much as possible since they can increase patients harm, discontent and healthcare costs.

INR testing (step 3) was omitted in $12 \%$ of patients. Although not mandated in all guidelines, ensuring INR levels are safe prior to surgery is likely beneficial in preventing adverse bleeding events related to the surgery.

The bridging decision (steps 4 and 5) was not in accordance with the guidelines in almost one in three patients. Furthermore, the postoperative performance was significantly lower in non-elective patients due to an overuse of bridging in low-risk patients. Emergency surgery settings with less time or priority for assessing the need for bridging anticoagulation might contribute to this. However, the 
Table 4 Perioperative anticoagulant management (PAM) practice characteristics and compliance for postoperative steps

\begin{tabular}{|c|c|c|c|c|}
\hline PAM step & $\begin{array}{l}\text { Elective surgery } \\
(n=184)\end{array}$ & $\begin{array}{l}\text { Non-elective surgery } \\
(n=75)\end{array}$ & P value* & $\begin{array}{l}\text { Total } \\
(\mathrm{n}=259)\end{array}$ \\
\hline \multicolumn{5}{|c|}{ 5. Apply or withhold postoperative bridging anticoagulation according to thromboembolic risk } \\
\hline Valid records, $n(\%)$ & $181(98.4)$ & $75(100)$ & - & $256(98.8)$ \\
\hline \multicolumn{5}{|c|}{ Applied bridging per thromboembolic risk strata, $\mathrm{n}(\%)$} \\
\hline Low & $30(29.4)$ & $16(48.5)$ & 0.044 & $46(34.1)$ \\
\hline Moderate & $16(59.3)$ & $6(54.5)$ & 0.79 & $22(57.9)$ \\
\hline High & $12(54.5)$ & $7(38.9)$ & 0.32 & $19(47.5)$ \\
\hline Unknown & $15(50.0)$ & $5(38.5)$ & 0.49 & $20(46.5)$ \\
\hline Compliance, \% (95\% Cl)† & $73.5(66.5$ to 80.5$)$ & 56.5 (44.1 to 68.8$)$ & 0.015 & 68.5 (62.3 to 74.8$)$ \\
\hline
\end{tabular}

6. Restart bridging anticoagulation, if ordered, 24 hours after surgery. Restart after 12 hours is allowable for high thromboembolic risk patientsł

\begin{tabular}{|c|c|c|c|c|}
\hline Applicable records (bridging used), n (\%) & $63(36.8)$ & $29(42.0)$ & & $92(38.3)$ \\
\hline Valid records, $\mathrm{n}(\%)$ & $63(100)$ & $29(100)$ & & $92(100)$ \\
\hline $\begin{array}{l}\text { Day of postoperative bridging (re)start, } \\
\text { median (IQR) }\end{array}$ & $1(0-1)$ & $1(1-2)$ & 0.09 & $1(0-1)$ \\
\hline Surgery day, n (\%) & $20(31.7)$ & $6(20.7)$ & - & $26(28.3)$ \\
\hline First day after surgery, n (\%) & $34(54.0)$ & $14(48.3)$ & - & $48(52.2)$ \\
\hline Second day after surgery, $\mathrm{n}(\%)$ & $4(6.3)$ & $4(13.8)$ & - & $8(8.7)$ \\
\hline Third day after surgery or later, $\mathrm{n}(\%)$ & $5(7.9)$ & $5(17.2)$ & - & $10(10.9)$ \\
\hline Compliance, \% (95\% Cl) & 60.7 (48.4 to 72.9$) \S$ & 51.7 (33.5 to 69.9$)$ & 0.42 & 57.8 (47.6 to 68.0$)$ \\
\hline \multicolumn{5}{|l|}{ 7. Restart VKA 24 hours after surgery $\ddagger$} \\
\hline Applicable records (VKA restarted), n (\%) & $161(94.2)$ & $66(95.7)$ & - & $227(94.6)$ \\
\hline Valid records, n (\%) & $136(84.5)$ & $62(93.9)$ & - & $198(87.2)$ \\
\hline $\begin{array}{l}\text { Day of postoperative VKA restart: median } \\
\text { (IQR) }\end{array}$ & $1(1-3)$ & $2(1-4)$ & 0.14 & $2(1-3)$ \\
\hline Surgery day, n (\%) & $13(9.6)$ & $4(6.5)$ & - & $17(8.6)$ \\
\hline First day after surgery, $\mathrm{n}(\%)$ & $57(41.9)$ & $22(35.5)$ & - & 79 (39.9) \\
\hline Second day after surgery, $\mathrm{n}(\%)$ & $18(13.2)$ & $8(12.9)$ & - & $26(13.1)$ \\
\hline Third day after surgery or later, $\mathrm{n}(\%)$ & $48(35.3)$ & $28(45.2$ & - & $76(38.4)$ \\
\hline Compliance, \% (95\% Cl) & 41.9 (33.6 to 50.2$)$ & 35.5 (23.6 to 47.4$)$ & 0.39 & 39.9 (33.1 to 46.7$)$ \\
\hline
\end{tabular}

Unless otherwise stated, all results are based on valid and applicable records only.

${ }^{*} \chi^{2}$ or Mann-Whitney $U$ test between elective and non-elective surgery populations.

tBased on valid records and records with known thromboembolic risk.

$\ddagger$ Records of patients who underwent second surgery were omitted (elective surgery $n=13$, non-elective surgery $n=6$ ).

$\S$ Records of patients with unknown thromboembolic risk and bridging restart at surgery day $(n=2)$ were omitted. Thromboembolic risk is required to determine compliance for these patients.

VKA, vitamin-K antagonist.

guidelines are originally aimed at elective patient populations, so our results for non-elective patients should be interpreted accordingly. Regardless of this, the identified difference warrants further investigation of postoperative PAM in non-elective surgery settings. Future guidelines should consider including a statement or recommendation for non-elective surgery patients to inform involved professionals.

Also, the use of subtherapeutic dose bridging in 50\% of patients was unexpectedly high given the explicit CBO 2008 recommendation to provide therapeutic dose bridging. Insufficient dose adjustments for bodyweight appeared to be contributing to this observation.

Most cases of non-compliance we observed, directly increase the risk for adverse events. Therefore, our study can indirectly explain some of the occurrences of anticoagulant-related adverse events that occur around surgery and are reported in other studies. ${ }^{143}$

\section{Quality improvement for PAM}

Our reliability findings inform the development of quality improvement measures for PAM. Since we evaluated the 


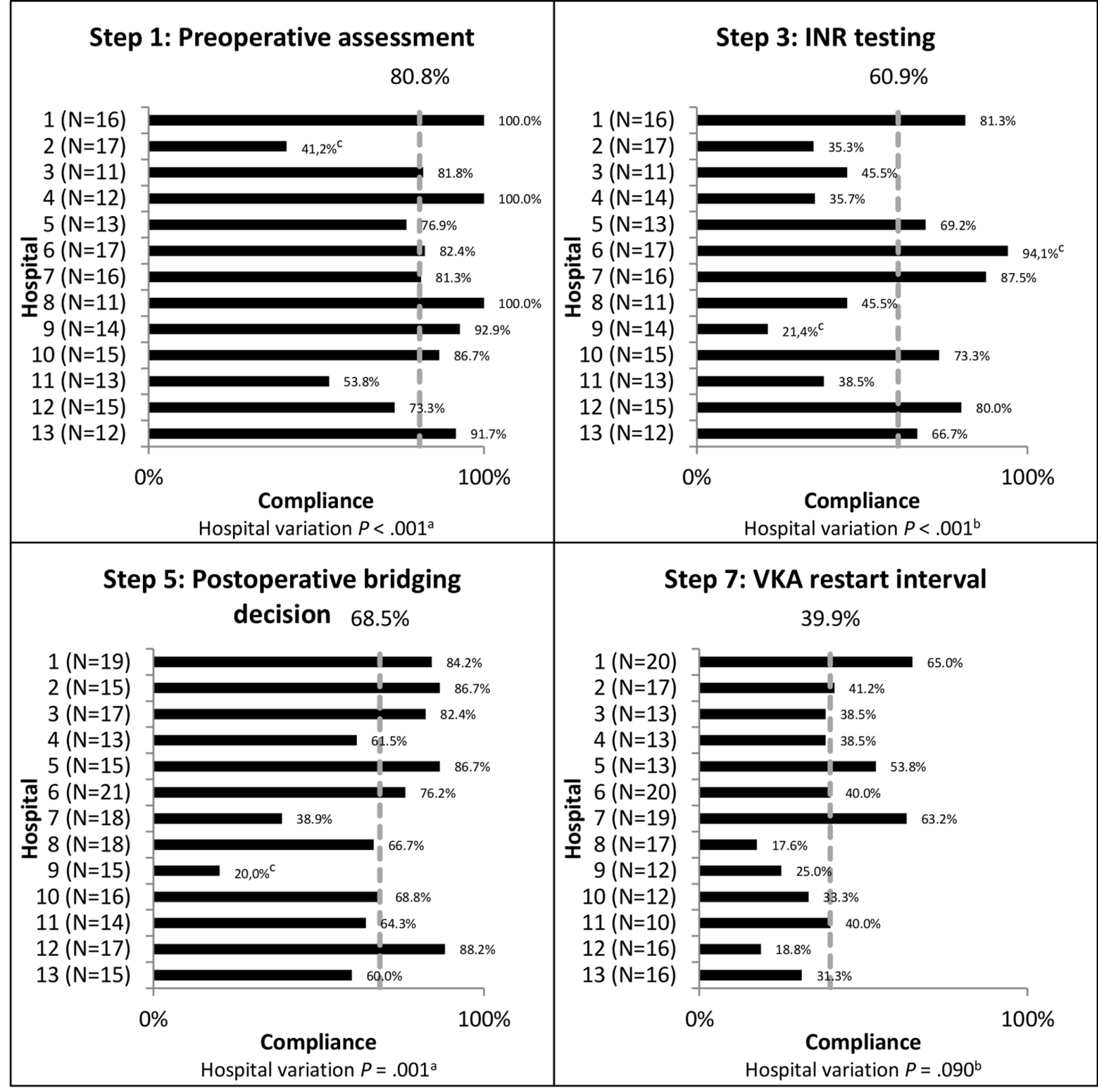

a Fisher's exact test

${ }^{b} \chi^{2}$ test

'Hospital's compliance is significantly different. (Post-hoc chi-square test: $P<.05$, Bonferroni correction applied)

Figure 1 Variation in compliance between individual hospitals for steps 1, 3, 5 and 7 . The dashed vertical line represents the population average. INR, international normalised ratio; VKA, vitamin-K antagonist.

hospitals' performance on the delivery of PAM as oppose to patient outcomes, process measures are most appropriate. Process measures reflect the delivery of care to patients and can inform us of the quality of this care. ${ }^{34} 35$ However, not all PAM steps with corresponding guideline recommendations will be as easily transformed in an effective process measure. For example, the decision whether to use bridging requires a large number of specific risk factors that have to be brought together and comprehended to anticipate on the most favourable outcome for the patient. This will be challenging to include in a single process measure. More straightforward candidates for PAM process measures are the timing of patient assessment or the preoperative INR testing. Supported by modern electronic health record and appointment data, evaluation of these steps should not be problematic. This is confirmed in our study by the amount of valid record entries for these two steps compared with other steps.

Another challenge for quality improvement involves the integrated care aspect of PAM. In the Netherlands, 52 AMS and 121 hospitals are present, ${ }^{36}{ }^{37}$ indicating heterogeneous service areas and resulting in multiple collaborations. A national integrated anticoagulant care standard aims to align involved healthcare providers and their responsibilities. ${ }^{28}$ However, a recent qualitative process analysis of preoperative PAM found a divergent 
practice pattern regarding the responsibilities of hospitals and AMS during preoperative PAM. Barriers in implementing guidelines at integrated care level, such as a lack of common governance, different logistics, medical oversight and funding, as described by Lang et al might be at work here. ${ }^{38}$ Implementing a shared responsibility for preoperative PAM might encourage the alignment of involved integrated care providers.

As a final point towards PAM quality improvement, it is worthwhile to note that new evidence for PAM in VKA patients is rapidly emerging. Guidelines are quickly complemented by new evidence, among which is evidence suggesting a relation between early onset $(<24$ hours) of postoperative bridging anticoagulation and major bleeding complications. ${ }^{39}$ Other evidence competes with the effectiveness of bridging anticoagulation. The BRIDGE (Perioperative Bridging Anticoagulation in Patients with Atrial Fibrillation) trial found that bridging did not reduce thromboembolic complications but increased the risk for bleeding complications compared with non-bridging. ${ }^{40}$ This trial was published in the last month of our patient inclusion (December 2015) so its suspected impact in terms of reduced bridging in atrial fibrillation patients had not been translated into clinical practice yet. Since a majority of our study population were atrial fibrillation patients and bridging low-risk patients was the most prevalent form of non-compliance, the BRIDGE trial findings might have positively influenced the overall bridging compliance from December 2015 onwards. What adds to this expectation is the Dutch national guideline update in April 2016, where the number of thromboembolic risk strata got limited to only two. Most of the patients in the moderate risk stratum are reassigned towards the lower risk stratum for which bridging is not recommended. ${ }^{31}$ These developments show that the evidence base is still subject to change rather than well established, posing an obstacle for quality improvement measure development. ${ }^{41}$ Furthermore, long guideline update intervals, delay new evidence to reach practice. ${ }^{42}$

\section{Strengths and limitations}

Our study has several strengths and limitations. First of all, the multicentre design with a representative sample of Dutch hospitals provides us with insight into current PAM practice in the Netherlands. Furthermore, the use of patient record data allowed us to evaluate the 'realworld' PAM practice without risking bias caused by study setting or observation.

At the same time, the dependency on routine patient record data has its own limitations. The PAM registration in the records was not always of high quality. This became apparent for the preoperative VKA interruption, details regarding preoperative bridging and documentation of postsurgical haemostasis. These were sometimes insufficiently documented. Although this probably introduced some bias to the compliance assessment for these two steps, it is not expected to change conclusions much due to the high prevalence of non-compliance in valid records.

Additionally, the specific agreements between hospitals and involved AMS regarding PAM cooperation were not available for this study. Therefore, we cannot rule out that some of our observed variation is attributable to between-hospital variation regarding such cooperations.

The voluntary participation of hospitals poses as another limitation. In total, 25 hospitals were invited to participate, of which only 13 accepted. Therefore, we cannot exclude the possibility of some selection bias at hospital level.

As a final limitation, we wish to acknowledge that no clinical outcomes were ascertained, preventing the establishment of a relationship with observed practice patterns.

\section{CONCLUSIONS}

Based on the unsatisfying compliance with most guideline recommendations, we can conclude that the reliability of the PAM process in the Netherlands is suboptimal. Additionally, PAM varies between hospitals and different dosages of bridging anticoagulation are used due to suboptimal adjustments for patients' weight. These findings confirm that standardised PAM is complex and not straightforward to implement in everyday practice. The observed non-compliance in many cases directly increases the risk of adverse events in individual patients.

Our study informs the development of process measures for PAM aimed at monitoring and quality improvement. Our PAM step approach can be used as a prelude to a future checklist for self-audits or standardisation of PAM documentation. However, several challenges have to be overcome also. Among these are: obtaining a stronger evidence base, reducing the knowledge to action gap and alignment of integrated care providers involved in PAM.

\section{Author affiliations}

${ }^{1}$ Department of Public and Occupational Health, Amsterdam Public Health Research Institute, Amsterdam UMC, Vrije Universiteit Amsterdam, Amsterdam, The Netherlands

${ }^{2}$ Netherlands Institute for Health Services Research (NIVEL), Utrecht, The Netherlands

${ }^{3}$ Departmentof Quality and Safety, Elisabeth-TweeSteden Ziekenhuis, Tilburg, The Netherlands

${ }^{4}$ Department of Thrombosis and Hemostasis, Leids Universitair Medisch Centrum, Leiden, The Netherlands

Acknowledgements We wish to acknowledge F.W.G. Leebeek from the Erasmus University Medical Center, T.P.W. Kamphuisen from the University Medical Center Groningen, S. Middeldorp from the Academic Medical Center Amsterdam and N.R. Bijsterveld from the Flevoziekenhuis in Almere for their contribution to this study as expert panel members. Additionally, we acknowledge J.W.R. Twisk from the VU University Medical Center for statistical advice and W. van der Schors, M. Tanaka and L.M. Bouma for assisting with the data collection.

Contributors MJM, NLD, MVH, MdB and CW designed the study and developed the study protocol. MVH chaired the expert panel and contributed to the study design. MJM and NLD organised the data collection. MJM collected the data. MJM performed statistical analyses. MJM and JFdG interpreted the results. MJM wrote the manuscript. All authors made critical revisions and approved the final version of the manuscript. 
Funding This work was supported by the Dutch Ministry of Healthcare Welfare and Sport.

Competing interests None declared.

Patient consent for publication Not required.

Ethics approval The study protocol was approved by the medical ethics committee of the VU University Medical Centre, Amsterdam, The Netherlands.

Provenance and peer review Not commissioned; externally peer reviewed.

Data sharing statement Data may be obtained from a third party and are not publicly available.

Open access This is an open access article distributed in accordance with the Creative Commons Attribution Non Commercial (CC BY-NC 4.0) license, which permits others to distribute, remix, adapt, build upon this work non-commercially, and license their derivative works on different terms, provided the original work is properly cited, appropriate credit is given, any changes made indicated, and the use is non-commercial. See: http://creativecommons.org/licenses/by-nc/4.0/.

\section{REFERENCES}

1. Penning-van Beest FJ, van Meegen E, Rosendaal FR, et al. Characteristics of anticoagulant therapy and comorbidity related to overanticoagulation. Thromb Haemost 2001;86:569-74.

2. Holbrook A, Schulman S, Witt DM, et al. Evidence-based management of anticoagulant therapy: Antithrombotic therapy and prevention of thrombosis, 9th ed: American college of chest physicians evidence-based clinical practice guidelines. Chest 2012;141:e152S-84.

3. Kirchhof P, Benussi S, Kotecha D, et al. 2016 ESC Guidelines for the management of atrial fibrillation developed in collaboration with EACTS. Eur Heart J 2016;37:2893-962.

4. La Regina M, Orlandini F, Marchini F, et al. Combined assessment of thrombotic and haemorrhagic risk in acute medical patients. Thromb Haemost 2016;115:392-8.

5. Hoonhout LH, de Bruijne MC, Wagner C, et al. Nature, occurrence and consequences of medication-related adverse events during hospitalization: a retrospective chart review in the Netherlands. Drug Saf 2010;33:853-64.

6. Morimoto T, Sakuma M, Matsui K, et al. Incidence of adverse drug events and medication errors in Japan: the JADE study. J Gen Intern Med 2011;26:148-53.

7. Damen NL, Baines R, Wagner C, et al. Medication-related adverse events during hospitalization: a retrospective patient record review study in The Netherlands. Pharmacoepidemiol Drug Saf 2017;26:32-9.

8. Marquet $\mathrm{K}$, Claes N, De Troy E, et al. A multicenter record review of in-hospital adverse drug events requiring a higher level of care. Acta Clin Belg 2017;72:156-62.

9. Vincent C, Burnett S, Carthey J. Safety measurement and monitoring in healthcare: a framework to guide clinical teams and healthcare organisations in maintaining safety. BMJ Qual Saf 2014;23:670-7.

10. Beadles CA, Hassmiller Lich K, Viera AJ, et al. A non-experimental study of oral anticoagulation therapy initiation before and after national patient safety goals. BMJ Open 2014;4:e003960.

11. Cantin A, Lahaie A, Odobasic B, et al. Evaluation of a continuous quality improvement program in anticoagulant therapy: Feasibility, satisfaction and perception. Can Pharm J 2016;149:352-61.

12. Barnes GD, Kline-Rogers $E$. Engaging with quality improvement in anticoagulation management. J Thromb Thrombolysis 2015;39:403-9.

13. Douketis JD, Spyropoulos AC, Spencer FA, et al. Perioperative management of antithrombotic therapy: Antithrombotic therapy and prevention of thrombosis, 9th ed: American college of chest physicians evidence-based clinical practice guidelines. Chest 2012;141:e326S-e50S.

14. Henriksen JN, Nielsen LP, Hellebek A, et al. Medication errors involving anticoagulants: Data from the Danish patient safety database. Pharmacol Res Perspect 2017;5:e00307.

15. Levine MN, Raskob G, Landefeld S, et al. Hemorrhagic complications of anticoagulant treatment. Chest 1998;114:511S-23.

16. Vongpatanasin W, Hillis LD, Lange RA. Prosthetic heart valves. $N$ Engl J Med 1996;335:407-16.

17. Siegal D, Yudin J, Kaatz S, et al. Periprocedural heparin bridging in patients receiving vitamin $\mathrm{K}$ antagonists: systematic review and meta-analysis of bleeding and thromboembolic rates. Circulation 2012;126:1630-9.
18. Kovacs MJ, Kearon C, Rodger M, et al. Single-arm study of bridging therapy with low-molecular-weight heparin for patients at risk of arterial embolism who require temporary interruption of warfarin. Circulation 2004;110:1658-63.

19. Douketis JD, Johnson JA, Turpie AG. Low-molecular-weight heparin as bridging anticoagulation during interruption of warfarin: assessment of a standardized periprocedural anticoagulation regimen. Arch Intern Med 2004;164:1319-26.

20. Douketis JD, Berger PB, Dunn AS, et al. The perioperative management of antithrombotic therapy. Chest 2008;133:299S-

21. Jaffer AK, Ahmed M, Brotman DJ, et al. Low-molecular-weightheparins as periprocedural anticoagulation for patients on long-term warfarin therapy: a standardized bridging therapy protocol. J Thromb Thrombolysis 2005;20:11-16.

22. Malato A, Saccullo G, Lo Coco L, et al. Patients requiring interruption of long-term oral anticoagulant therapy: the use of fixed subtherapeutic doses of low-molecular-weight heparin. J Thromb Haemost 2010;8:107-13.

23. Pengo V, Cucchini U, Denas G, et al. Standardized low-molecularweight heparin bridging regimen in outpatients on oral anticoagulants undergoing invasive procedure or surgery: an inception cohort management study. Circulation 2009;119:2920-7.

24. Steib A, Mertes PM, Marret E, et al. Compliance with guidelines for the perioperative management of vitamin K antagonists. Thromb Res 2014;133:1056-60.

25. Bruno M, Marengo A, Elia C, et al. Antiplatelet and anticoagulant drugs management before gastrointestinal endoscopy: do clinicians adhere to current guidelines? Dig Liver Dis 2015;47:45-9.

26. Eijgenraam $\mathrm{P}$, ten Cate $\mathrm{H}$, ten Cate-Hoek AJ. Practice of bridging anticoagulation: guideline adherence and risk factors for bleeding. Neth J Med 2014;72:157-64.

27. Lum DJ, Ross PA, Bishop MA, et al. Evaluation of a Standardized Perioperative Management Protocol in the Adult Hematology Anticoagulation Management Service. Ann Pharmacother 2017;51:1077-83.

28. Landelijke Standaard Ketenzorg Antistolling versie 2. In: Specialisten $K M$, ed 2014

29. Diagnostiek R. Preventie en Behandeling van Veneuze Tromboembolie en Secundaire Preventie Arteriële Trombose. CBO (Kwaliteitsinstituut voor de Gezondheidszorg): Utrecht, 2008.

30. Ansell J, Hirsh J, Poller L, et al. The pharmacology and management of the vitamin K antagonists: the Seventh ACCP Conference on Antithrombotic and Thrombolytic Therapy. Chest 2004;126(3 Suppl):204s-33.

31. Richtlijn Antitrombotisch Beleid: Integraal Kankercentrum Nederland Kennisinstituut Medisch Specialisten 2016.

32. Flaker GC, Theriot P, Binder LG, et al. Management of Periprocedura Anticoagulation: A Survey of Contemporary Practice. J Am Coll Cardiol 2016;68:217-26.

33. Moesker MJ, Schutijser B, de Groot JF, et al. Occurrence of Antithrombotic Related Adverse Events in Hospitalized Patients: Incidence and Clinical Context between 2008 and 2016. J Clin Med 2019;8:839.

34. Donabedian A. An introduction to quality assurance in health care: Oxford University Press, 2002

35. Types of Quality Measures. Rockville, MD: Agency for Healthcare Research and Quality, 2011. updated July 2011. http://www.ahrq. gov/professionals/quality-patient-safety/talkingquality/create/types. html. (Accessed 19 Jul 2018).

36. Samenvatting Medische Jaarverslagen 2015. Federatie van Nederlandse Trombosediensten FNT, 2016.

37. Hospital Care, Regional and International: National Institute for Public Health and the Environment. 2018 https://www.volksgezondh eidenzorg.info/onderwerp/ziekenhuiszorg/regionaal-internationaal/ locaties\#bronverantwoording (Accessed 30 apr 2018).

38. Lang ES, Spaite DW, Oliver ZJ, et al. A national model for developing, implementing, and evaluating evidence-based guidelines for prehospital care. Acad Emerg Med 2012;19:201-9.

39. Tafur AJ, McBane R, Wysokinski WE, et al. Predictors of major bleeding in peri-procedural anticoagulation management. $J$ Thromb Haemost 2012;10:261-7.

40. Douketis JD, Spyropoulos AC, Kaatz S, et al. Perioperative Bridging Anticoagulation in Patients with Atrial Fibrillation. N Engl J Med 2015;373:823-33.

41. Chassin MR, Loeb JM, Schmaltz SP, et al. Accountability measuresusing measurement to promote quality improvement. $N$ Engl J Med 2010;363:683-8.

42. Graham ID, Logan J, Harrison MB, et al. Lost in knowledge translation: time for a map? J Contin Educ Health Prof 2006;26:13-24. 